\title{
Fibrilación auricular: mantener el ritmo sinusal tiene similares resultados a controlar la frecuencia cardíaca
}

A comparison of rate control and rhythm control in patients with atrial fibrillation. The atrial fibrillation follow-up investigation of rhythm management (AFFIRM). NEJM 2002;347:1825-33

\section{Objetivo}

Evaluar si la estrategia de mantenimiento del ritmo sinusal en pacientes con fibrilación auricular (FA) es superior a la estrategia de control de la frecuencia cardiaca más anticoagulación.

Diseño

Ensayo clínico randomizado, abier to, con un seguimiento promedio de 3,5 años

Lugar

Estudio multicéntrico realizado en 213 centros de Estados Unidos y Canadá.

\section{Pacientes}

Fueron incluidos 4060 pacientes (61\% hombres) con FA recurrente, mayores de 65 años (edad media $70 \pm 9$ ) o con otro factor de riesgo embólico, sin contraindicaciones de anticoagulación y que, a juicio del médico tratante, requir ieran tratamiento para su FA. Intervención

Se randomizó a los pacientes según la estrategia de tratamiento de la FA a control de ritmo (drogas antiarrítmicas a elección del médico tratante y/o cardioversión eléctrica) versus control de frecuencia (con beta bloqueantes, digoxina y/o bloqueantes cálcicos, solos o en combinación).

En ambos grupos se permitieron terapéuticas "no farmacológicas" (ablación por radiofrecuencia, operación de "Maze", implante de marcapasos) en los casos necesarios. El rango de anticoagulación fue RIN 2 a 3 (obligatoria en el grupo control de frecuencia y optativa en el grupo control de ritmo).

\section{Medición de los resultados principales}

El punto final primario fue mortalidad total. Puntos secundarios fueron muerte, accidente cerebrovascular (ACV) discapacitante, encefalopatía anóxica, sangrado mayor y paro cardíaco.

\section{Resultados principales}

Hubo 356 muertes en el grupo control de ritmo y 310 muertes en el grupo control de frecuencia (mortalidad a cinco años $23,8 \%$ vs. $21,3 \%$, RR 1,15 (95\% IC 0,99 a 1,34, $p=0,08)$. Más pacientes en el grupo control de ritmo fueron hospitalizados $(80 \%$ vs. $73 \%$, $p<$ 0,001). Los ACV embólicos (77 pacientes en el grupo control de frecuencia vs 80 en el grupo control de ritmo) ocurrieron luego de la suspensión de la anticoagulación o con anticoagulación insuficiente. Los índices de calidad de vida fueron similares en ambos grupos.

\begin{tabular}{l|l|l|l} 
& $\begin{array}{l}\text { Control de la frecuencia } \\
(\mathbf{n}=2027)\end{array}$ & $\begin{array}{l}\text { Control del ritmo } \\
(\mathbf{n}=2033)\end{array}$ & Valor de $\mathbf{P}$ \\
\hline Mortalidad total & $310(15,3 \%)$ & $356(15,5 \%)$ & 0,08 \\
\hline ACV isquémico & $77(3,7 \%)$ & $80(3,9 \%)$ & 0,79 \\
\hline Internaciones & $1220(60,2 \%)$ & $1374(67,6 \%)$ & 0,001 \\
\hline
\end{tabular}

\section{Conclusiones}

La estrategia de mantenimiento del ritmo sinusal en pacientes con FA no ofrece ventajas en sobrevida con respecto a la estrategia de control de frecuencia. La estrategia de control de frecuencia tiene la ventaja potencial de evitar los efectos adversos de las drogas antiarrítmicas. La anticoagulación debe continuarse en este grupo de pacientes de alto riesgo embólico.

Fuente de financiamiento:National Heart, Lung and Blood Institute de USA y laboratorio Wyeth-Ayerst (amiodarona).

\section{Comentario}

Este estudio demuestra que la simple estrategia de control de la frecuencia cardíaca y anticoagulación en pacientes con FA es similar (o quizás mejor) que la estrategia, más compleja, de mantener el ritmo sinusal (denominado control de ritmo) en una población añosa y de alto riesgo embolígeno. Los resultados del estudio AFFIRM son congruentes con otro estudios publicado en la misma revista (Rate Control versus Electrical Cardioversion for Persistent atrial Fibrillation) en donde se randomizaron 522 pacientes en un diseño muy similar ${ }^{1}$. En este estudio europeo tampoco se observaron diferencias significativas entre las dos estrategias de tratamiento de la FA.

A primera vista el estudio AFFIRM fue bien diseñado y realizado, pero habría que remarcar varios problemas metodológicos.

1. La FA es una arritmia muy dinámica, con frecuentes recurrencias en algunos pacientes y gran estabilidad en la respuesta a los tratamientos en otros. Los tratamientos utilizados en estos pacientes son también muy variables y dinámicos. Por este motivo, del grupo inicialmente randomizado a control de ritmo, el $38 \%$ de los pacientes se cruzó a la otra estrategia (control de frecuencia) con la consiguiente perdida del supuesto "beneficio" de la mantención del ritmo sinusal durante los años de seguimiento, lo que le resta claridad a las conclusiones.

2. La eficacia del tratamiento antiarrrítmico para mantener a los pacientes en ritmo sinusal fue muy baja (aproximadamente el $40 \%$ de los pacientes en el grupo control de ritmo no mantuvo el ritmo sinusal a los cinco años de seguimiento). Los resultados tal vez habrían sido otros (favoreciendo a la estrategia de control de ritmo) si se hubiera comparado la evolución de los pacientes que "realmente" mantuvieron el ritmo sinusal a lo largo del seguimiento, en lugar de analizarlos junto con el $40 \%$ de los pacientes que no sólo no mantuvieron el ritmo sinusal, sino que además estuvieron expuestos a los efectos adversos de las drogas antiarrítmicas. Las nuevas estrategias "no farmacológicas" para tratar la FA (ej. ablación por radiofrecuencia de las venas pulmonares) tal vez puedan mejorar estos resultados.

3. Había que destacar que la edad media de la población fue de 70 años, por lo que los resultados de estos estudios solo deberían considerarse en la población añosa. La población de pacientes jóvenes con FA habitualmente son mas sintomáticos, mejorando en ellos la calidad de vida en forma significativa con el mantenimiento del ritmo sinusal.

4.Finalmente, en ambos estudios se incluyeron pacientes con FA mayoritariamente refractaria al tratamiento y recurrente (con cardioversión eléctrica previa) por lo que estos resultados no serían aplicables a pacientes con un primer episodio de arritmia. En estos pacientes no debería negarse la posibilidad de una reversión a ritmo sinusal, sea ésta por medio de cardioversión eléctrica o farmacológica, sobre todo en un primer o segundo episodio de arritmia, ya que más de la mitad de ellos van a mantener el ritmo sinusal en el seguimiento alejado ${ }^{2,3}$.

\section{Conclusión del comentador}

Hasta que no mejoren las herramientas disponibles para el tratamiento de la FA y podamos brindar medidas de tratamiento más eficaces y definitivas, en pacientes añosos (> 65 años) con FA recurrente, el control apropiado de la frecuencia cardíaca y una anticoagulación adecuada no perjudica su evolución.

\section{Dr. Marcelo Helguera [ Servicio de Cardiología, Hospital Italiano de Buenos Aires ]}

\section{Referencias}

1. Van Gelder et al. for the rate control versus electrical cardioversion for persistent atrial fibrillation Study Group. A comparison of rate control and rhythm control inpatients with recurrent persistent atrial fibrillation.N Engl J Med 2002, 347:1834-40

2.Falk.Management of atrial fibrillation, radical response or modest modification.N Engl J Med 2002, 347:1883

3.Fuster V, Ryden LE, Asinger RE et al.AHA/ACC/ESC guidelines for the management of patients with atrial fibrillation executive summary American Heart Association, American College of Cardiology, European Society of Cardiology (Comitee to develop guidelines for the management of patients with atrial fibrillation) developed in association with the North American Society for pacing and electrophysiology J Am Coll Cardiol 2001;38:1231-1266. 